\title{
Analysis of wake surfing benefits using a fast unsteady vortex lattice method
}

\author{
Dominique Fleischmann* and Mudassir Lone ${ }^{\dagger}$ \\ Cranfield University, Cranfield, MK43 OAL, United Kingdom
}

\begin{abstract}
The computer simulation framework Flexit is used to analyse the fuel economy benefit of aircraft wake surfing. Wake surfing involves multiple aircraft flying in close formation during cruise conditions to reduce overall induced drag and improve overall fuel efficiency. The aircraft fly in echelon such that the kinetic energy lost in vortices generated by the lead aircraft can be partially recovered by the following aircraft flying in regions of the wake where induced velocities have an upwardly directed vertical component. We build on recent theoretical and flight test work by developing a medium fidelity methodology using Flexit for predicting potential performance benefits of wake surfing. We present results from a specific systematic parametric study that corresponds to a series of recent flight tests with two C-17 transport aircraft to demonstrate the methodology and predict the fuel savings that can be obtained by different arrangements of aircraft in a wake surfing formation. The predictions are compared with the flight test data and the trends observed in our simulations agree with the trends of the full scale tests.
\end{abstract}

\section{Introduction}

Today's push for rapid and responsive operational analysis has highlighted the need for tools that are computationally cheap and technically versatile. These tools need to allow the broad exploration of operational parametric spaces and also provide insight into the physics governing the dynamics of the system being considered. Wake surfing is an example of a technical field where such a tool is needed. We begin by giving an overview of the detailed and thorough research work into wake surfing conducted by DARPA, AFRL, Boeing, the University of Toronto and Ryerson University (see references [1], [2], [3], [4] and [5]) over the past decade.

The main focus of the paper is to present a new medium fidelity methodology for prediction of potential fuel consumption performance benefits from wake surfing. We demonstrate the approach by presenting the results of a set of calculations performed using this methodology for a pair of C-17 military transport aircraft flying straight and level in formation. This configuration closely matches the flight tests performed as part of the SAVE 1 flight trials conducted in October 2012 and July 2013 (see [6]), and allows us to directly compare our predictions with the results of the flight trials. New and bespoke algorithm development and modelling work is presented that we believe improves the quality, value and confidence in the body of knowledge that is emerging to support the concept of wake surfing.

The authors are aware of the experimental and mathematical modelling work conducted on the current topic by DARPA, AFRL and Boeing since 2007, and outlined in references [2], [6], [7], [3] and [4]. This previous work was conducted under the NASA Surfing Aircraft Vortices for Energy project. We also acknowledge work being conducted at Ryerson University and the University of Toronto in vortex modelling (see for example [5]). Here we attempt to build on the existing material in our new work.

We present a highly versatile tool for wake surfing analysis called Flexit and we use it to perform time domain nonlinear inviscid simulations of the aerodynamic interaction between different aircraft flying in formation. Once the benefits in reducing drag have been predicted, we use the standard Simulink ${ }^{\circledR}$ Turbofan Toolbox (see [8]) to predict the resulting fuel saving.

\section{Physical mechanism of wake surfing benefit}

Figure 1 presents a diagram illustrating the mechanism by which a reduction in drag $\left(\bar{T}_{i}\right)$ is experienced by a trailing aircraft due to flight in the upwash from a lead aircraft. The symbol $V_{\infty}$ denotes the far field free stream constant velocity vector and the symbol $\bar{V}$ denotes the velocity vector in the near field of the aircraft under study that includes the

\footnotetext{
*Visiting Fellow, Centre for Aeronautics, School of Aerospace, Transport \& Manufacturing, d.s.fleischmann@cranfield.ac.uk

†Senior lecturer, Centre for Aeronautics, School of Aerospace, Transport \& Manufacturing, m.m.lone@cranfield.ac.uk
} 
contribution due to the wing downwash. The actual near field velocity vector experienced by the aircraft under study including the component associated with the upwash from the leading aircraft is denoted $\bar{V}_{e}$. The lift force for the aircraft under study flying in isolation (without the lead aircraft) is directed parallel to the vector $\bar{L}$. When the lead aircraft upwash is taken into account, the lift force is parallel to the vector $\bar{L}_{e}$, and it is this forward rotation of the lift vector that leads to a smaller induced drag. A follower aircraft can produce the same lift as a lead aircraft, but at a smaller angle of attack, and this too contributes to a reduction of induced drag, and hence better fuel economy.

It is important to emphasise that just as benefit can be obtained by flying in a region of upwash left behind in the wake of a lead aircraft, so a disadvantage can be experienced by erroneously flying in a region of downwash left behind.

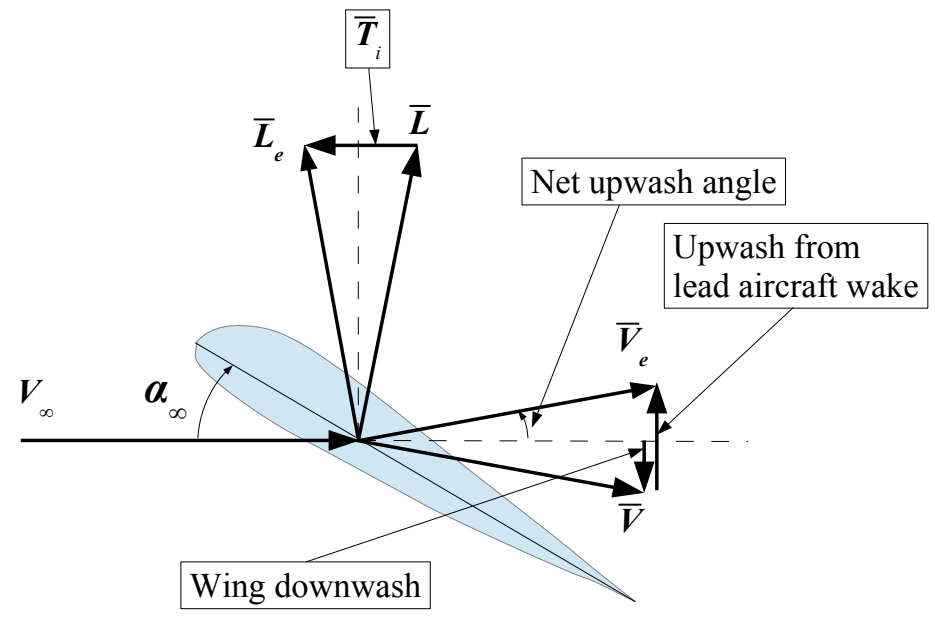

Fig. 1 Mechanism by which the benefits of wake surfing are felt by a trailing aircraft.

\section{Cost function}

In an overall cost-benefit analysis, the aim of wake surfing is to minimise the total cost of fuel used by all the aircraft in the wake surfing formation compared with the total cost of fuel used by the same aircraft, each flying in isolation at their individual optimum speeds and altitudes (in standard ISO atmospheric conditions). The cost function to be minimised is therefore

$$
C=\frac{F_{\text {formation }}}{F_{\text {individual }}}
$$

where the $F$ quantities are the total masses of fuel used (in kilograms) defined as

$$
F_{\text {formation }}=\sum_{n=1}^{N}\left(f_{\text {formation leg }}^{n}+f_{\text {outbound detour }}^{n}+f_{\text {inbound detour }}^{n}\right) \text {, }
$$

and

$$
F_{\text {individual }}=\sum_{n=1}^{N} f_{\text {ideal route }}^{n} .
$$

where $N$ is the total (fixed) number of aircraft in the wake surfing formation, and each aircraft is indexed by the symbol $n$. A value of $C$ less than 1 therefore indicates a net fuel cost benefit associated with wake surfing. 
The function $f_{\text {formation leg }}^{n}$ is the total mass of fuel (measured in kilograms) consumed by the $n^{\text {th }}$ aircraft in the formation during the formation leg of the journey. The function $f_{\text {outbound detour }}^{n}$ is the total mass of fuel consumed by the $n^{t h}$ aircraft during any detour required to join the formation. This may include loitering flight while waiting for other aircraft to arrive and/or flight to a rendez-vous point from a remote location. Similarly, the function $f_{\text {inbound detour }}^{n}$ is the total mass of fuel consumed by the $n^{t h}$ aircraft in the formation after leaving the formation to rejoin the individual route to the destination.

This cost function assumes a single type of fuel across all aircraft in a wake surfing operation, (purchased at a single fixed price per kilogram).

To simplify the analysis presented in this paper, and to remove the complications with modelling routes and loiter times, we consider here the simpler cost function given by

$$
C=\sum_{n=1}^{N}\left(\frac{f_{\text {formation }}^{n}}{f_{\text {individual }}^{n}}\right)
$$

where $f_{\text {formation }}^{n}$ is the mass of fuel burnt per second of the $n^{\text {th }}$ aircraft when flying in the formation and where $f_{\text {individual }}^{n}$ is the mass of fuel burnt per second of the $n^{\text {th }}$ aircraft when flying in isolation at the same altitude and speed and atmospheric conditions as the formation. Note that the altitude and speed of the formation may not be the optimum condition for each individual aircraft. This is particularly true when considering a heterogenous formation comprised of aircraft of different types and loading conditions.

We recognise that the weight of each aircraft will change during a formation leg (particularly if the leg is long), and as the weights of the individual aircraft reduce, the optimum arrangement of aircraft in the formation, the optimum speed of the formation and the optimum altitude of the formation may change. For the purpose of this study however, we assume that the weights of the individual aircraft (and their arrangement in the formation) and the speed and altitude of the formation itself remain constant during the formation leg.

It is recognised that in practice a single wake surfing operation may involve individual aircraft or groups of aircraft joining and/or leaving the formation, and changing their position in the formation during the operation. For the purposes of the simulations presented here, we consider only fixed formations where the number and arrangement of the aircraft is fixed.

\section{Overview of mathematical model}

At Cranfield University, we routinely use the computational framework Flexit to perform time-domain unsteady aeroelastic analysis of aircraft during the early design stages (see reference [9]). The Flexit framework combines the Unsteady Vortex Lattice Method (UVLM) for prediction of aerodynamic forces and moments, with a finite difference approximation to the Euler-Bernoulli partial differential equation and a finite element torsion model to predict the structural deformation in response to aerodynamic and interial loads. For this study we have disabled the structural deformation part of the algorithm and we assume that each aircraft in the wake surfing formation is rigid. Good descriptions of UVLM fundamentals can be found in references [10] and [11]. The algorithm design and software implementations in the Flexit framework include Rankine vortices (as opposed to Biot-Savart vortices) and much of the computationally intensive processing is performed on GPU using NVIDIA CUDA (see reference [12]). In addition, a more rigorous approach has been adopted for the computation of bound panel forces, using a first-principles Lorentz formulation.

For the computations described in this paper we only consider the aerodynamic behaviour of the aircraft main lifting surfaces, and for the $\mathrm{C}-17$ design considered, the main lifting surfaces comprise the main wing and horizontal tail-plane.

\section{A. Applicability of the mathematical model}

Before proceeding, it is important to note the domains of applicability of the UVLM and to check that application of the method to the aircraft in the wake surfing studies are valid. The UVLM uses potential flow theory, and so assumes that fluid viscous forces are insignificant compared with inertia forces. A dimensional analysis of the governing equations indicates that this is the case for high Reynolds number attached flows. For an aircraft wing the Reynolds number is given by

$$
R e=\frac{v L}{v}
$$


where $v$ is the aircraft velocity, $L$ is the wing chord length, and $v$ is the kinematic viscosity. In the case of the C-17, the lowest Reynold's number occurs at the main wing tip where the chord length is lowest. At this position on the wing we have a Reynolds number of approximately $1.2 \times 10^{6}$. This large value of $R e$ suggests that the flow over the wing aerofoil section is turbulent, and that viscous forces are small in comparison to inertia forces.

The UVLM also assumes incompressible flow, and so can only be applied to low speed flows significantly less than the speed of sound. The limiting case for this criteria is the speed of the flow at the main wing, where the cruise inflow airspeed is approximately $227 \mathrm{~m} / \mathrm{sec}$. Using a speed of sound at the operating altitude and temperature of $320 \mathrm{~m} / \mathrm{sec}$, and using a main wing sweep angle of 20 degrees we obtain a Mach number of approximately $0.7 \times \cos (20)=0.6578$. Taking these Reynolds number and Mach number values, together with the assumption that the flow remains attached, we see that the C-17 falls within the range of applicability of the UVLM.

At this point, the reader should note that at high subsonic speeds it is necessary to include some form of empirical correction to take account of compressibility effects. Flexit implements the Prandtl Glauert correction as described in [13].

\section{Flexit software implementation and performance}

The Flexit computational framework comprises a total of seven 64 bit executables written from scratch in $\mathrm{C}++$ that run under Windows 7 and Windows 10. Six of these programs are used to perform pre- and post-processing and the seventh, itself called Flexit, actually does the aero-elastic computations. The Flexit program can be run interactively with a graphical user interface or as a batch program from the command line. Figure 2 presents a screenshot of the Flexit program graphical user interface.

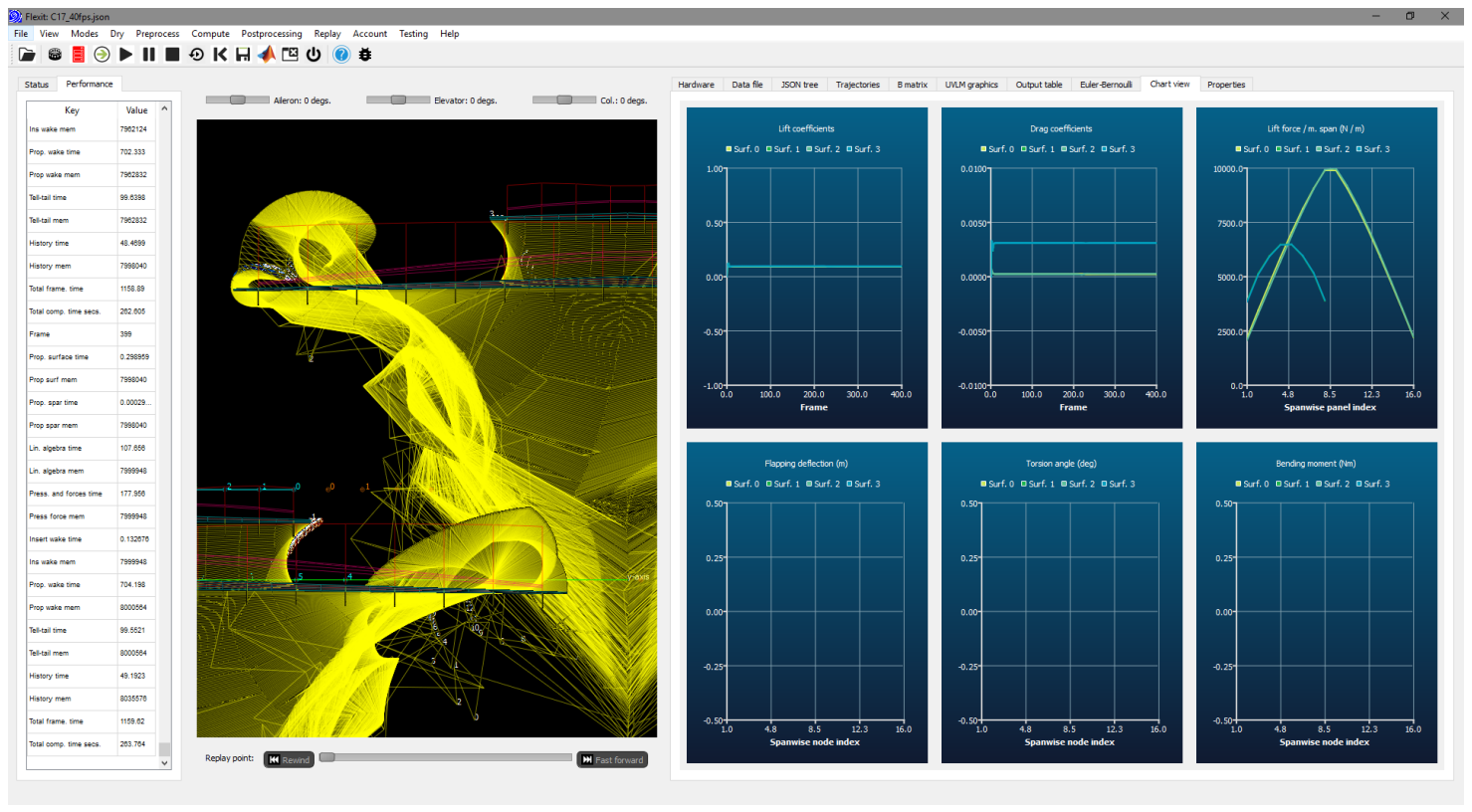

Fig. 2 Flexit screenshot

An analysis work-flow consists of the user running each program in a prescribed sequence, being led through the process of defining the lifting surface geometry, describing the properties and connectivity of the Euler-Bernoulli beams, describing the kinematic motion of the lifting surfaces, actually doing the aeroelastic analysis, and finally post-processing the results. Data output from the first program forms the input to the next, and so on. The Javascript Simple Object Notation (JSON) file format is used throughout. As well as the JSON files, functionality is also implemented to output data from the Flexit $\mathrm{C}++$ program as Matlab ${ }^{\circledR}$ script files with data arrays and script commands automatically included. Many of the figures in this paper have been prepared using this functionality.

The Flexit program performs the computationally intensive processing of the aeroelastic analysis. Most of the processing is concerned with doing the UVLM computations, and the most intensive part of this is concerned with computing the flow induced by the panels of known vorticity that have been shed into the wake. Fortunately the 
theory is amenable to parallel computation, and we have exploited this property to implement the algorithms in parallel on the GPU using the NVIDIA CUDA technology. In this way many thousands of computations are arranged to execute in parallel so that the overall elapsed time can be reduced by several orders of magnitude over a conventional CPU implementation. When the problem size is relatively small (when the number of panels is less than 2500, say) implementation on the GPU effectively reduces the problem from $O\left(N^{2}\right)$ to $O(N)$. Each analysis case presented in this paper involves computation over hundreds of UVLM time steps with up to 1000 bound panels and over 10,000 free wake panels. On a Power PC workstation with a single NVIDIA GeForce GTX 1080 GPU with relatively modest performance, typical simulations take approximately 180 seconds of wall-clock time to complete.

Figure 3 presents a graph from a typical simulation performed during the preparation of this paper on the Power PC workstation with an Intel ${ }^{\circledR}$ Core $^{\circledR}$ i7-7800X CPU and a single NVIDIA GeForce GTX 1080 GPU. It shows the wall-clock time for each computational frame, and the accumulated wall-clock time as the simulation progresses. The increases in frame execution time at frames 127 and 173 are related to GPU bandwidth. The spike at frame 60 is related to the fact that the program is sharing resources in Windows 10 pre-emptive operating system. As the number of wake panels increases, the number of GPU computations required exceeds the number of available parallel streams, and an extra tranche of GPU computations becomes necessary.

Figure 4 presents a corresponding area chart indicating the wall-clock time for each of the major computational tasks within a frame. From this figure it is clear that it is the free wake propagation computation that is largely responsible for this increase in overall execution time.

Figures 3 and 4 are significant, since they indicate that each computational frame is dominated by the UVLM computations. Despite the UVLM computation being $O\left(N^{2}\right)$, for a given number of computational tranches, the frame time only increases by $O(N)$. This is a direct consequence of implementing the algorithm in parallel using the GPU.

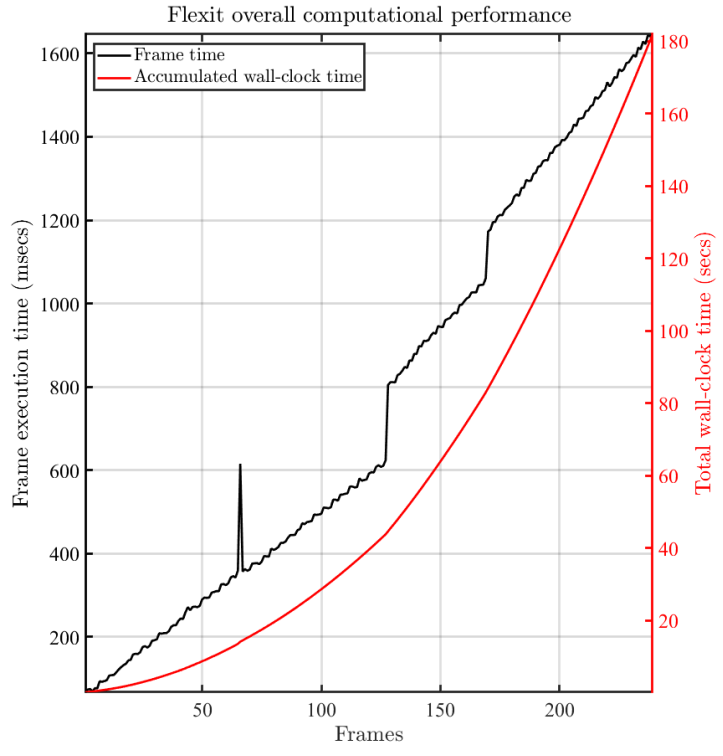

Fig. 3 Overall computational performance

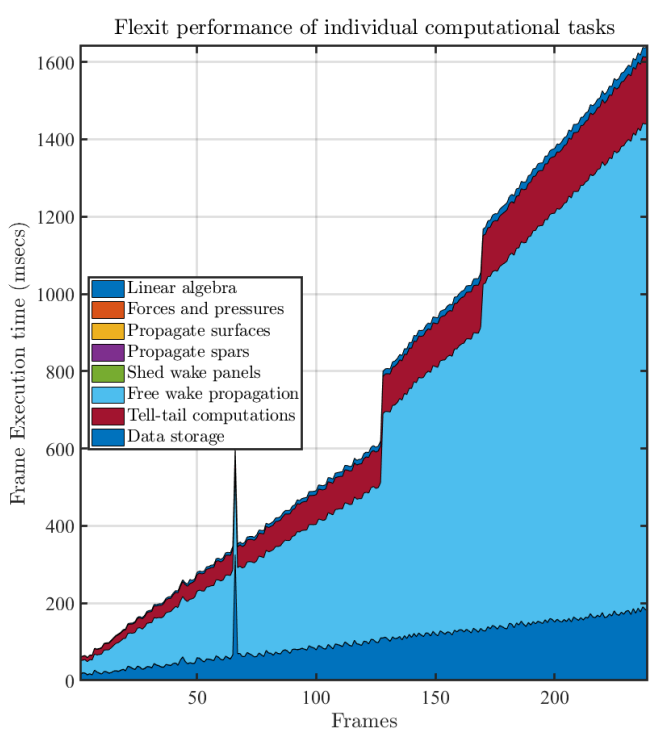

Fig. 4 Performance of individual computational tasks

\section{Parametric study}

\section{A. Assumptions}

To simplify the assessment of the relative merits of different formations, we assume that both aircraft use the same turbo-fan engines, and the same fuel purchased at the same price. We also assume that the weights of the individual aircraft and the formation airspeed and altitude remain constant during the formation leg.

As indicated in section [III above, we consider the sum of the fuel consumed during a straight and level formation leg itself (and ignore the outbound detour and the inbound detour). 
Vortex core size is only relevant for empirical methods. In UVLM with a Rankine vortex formulation, the vortex core radius is only implemented to avoid the core singularities associated with each of the many thousands of short vortex line segments. The value chosen for the Rankine core radius is typically $2 \mathrm{or} 3 \mathrm{~cm}$. In the UVLM method, the structure of the vortex core evolution is accounted for by the free motion of the many thousands of short vortex line segments of individually scaled vortex strengths under mutual influence. It is therefore not meaningful to define or impose another vortex core size in the UVLM method, and we avoid explicit definition of the of vortex core size in this work.

Free wake geometry (as opposed to a priori fixed wake geometry) is considered for the study. We ignore the effect of wind and atmospheric turbulence. Flexit is capable of including full aeroelastic behaviour. However as described above, we ignore these effects here. While there will be fuel economy penalties associated with correcting roll, pitch and yaw disturbances in the following aircraft, these are not considered, and may be included in future work.

It is recognised that for the best value of the cost function $C$, the lead aircraft in the formation may be forced to fly at a non-optimum airspeed and altitude. This may result in a higher fuel consumption than it would have when flying individually.

\section{B. Methodology}

The parametric study involves computing the reduction in drag as a result of flight in the upwash part of the wake of a lead aircraft, and then the computation of the reduction in fuel consumption that results. The reduction in drag comes about because the follower aircraft can generate the lift they require at a smaller angle of attack than they would while flying alone. To find the new reduced angle of attack requires an iterative approach. The methodology adopted for each case in this study is illustrated in figure 5 .

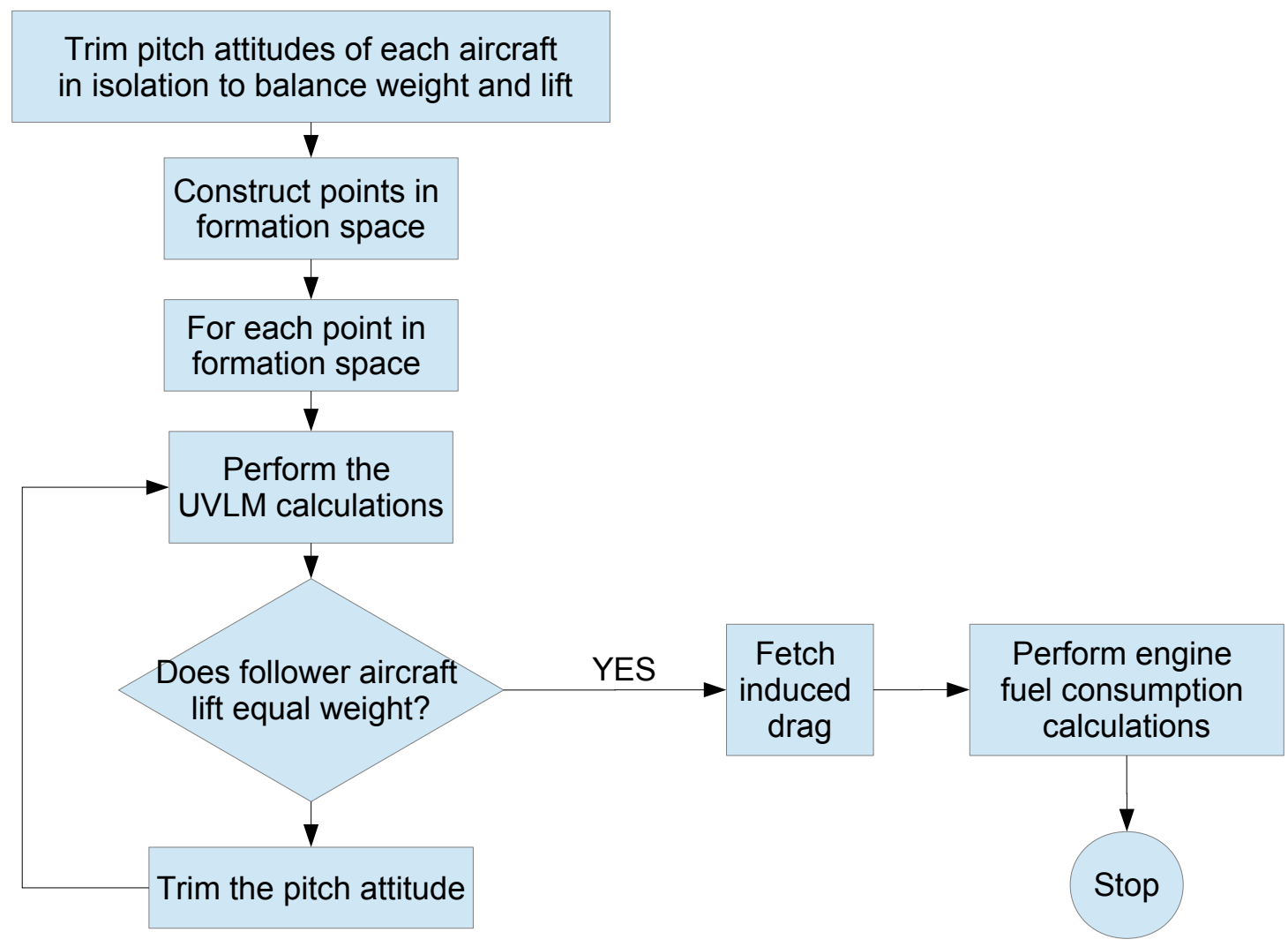

Fig. 5 Methodology adopted for each case in the parametric study 
The loop shown between "Trim the pitch attitude" and "Perform the UVLM calculations" is a manual process of trimming each of the follower aircraft. Since the UVLM calculations take a very short time this process is not very arduous.

\section{Planform geometry}

A study has been performed to predict the wake field far behind a simple geometrical model of a C-17 aircraft using the Flexit UVLM framework (version 0.6.001). Figure 6 presents the main wing and horizontal tail plane planform panel geometry that was used. This geometry is a highly simplified representation of the actual C-17 lifting surface geometry.

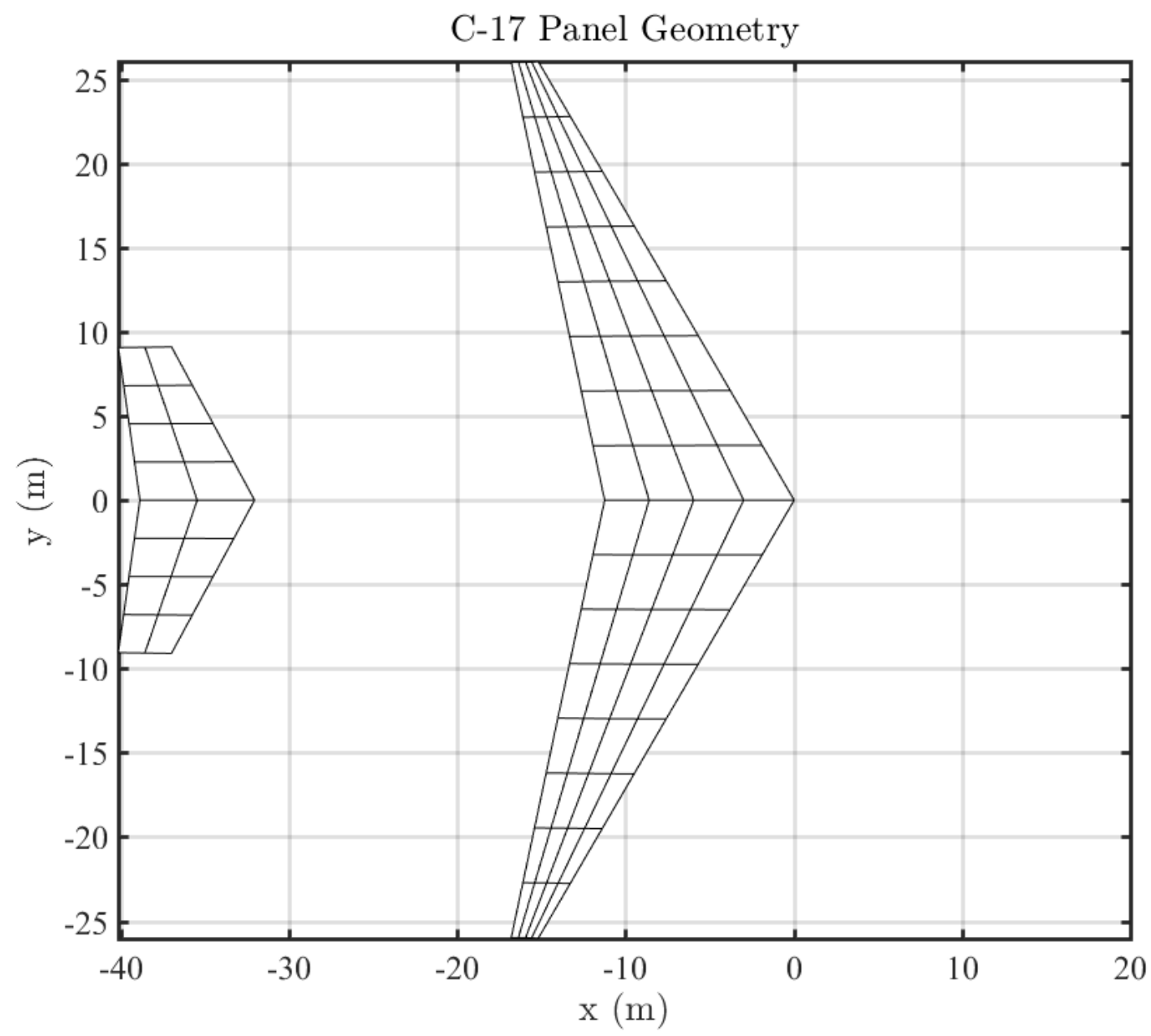

Fig. 6 UVLM panel geometry used for the two C-17 aircraft in the SAVE1 configuration

\section{C-17 homogeneous parametric study at SAVE I condition}

A parametric study for a formation consisting of two C-17 military transport aircraft is considered here. A simulation of a single follower aircraft and a single leader aircraft has been completed using Flexit UVLM (version 0.6.001). The aim of this study is to replicate the operational conditions encountered during the SAVE I flight trials conducted in 2012-13 and described in [6].

We compare fuel savings predicted by our method with those achieved in flight and indicated in figures 32 and 33 of reference [6]. Unfortunately the method of construction of figure 33 is not explicitly explained in the paper, so we 
naturally assume that the contour plot has been derived from the flight test data.

This study was conducted for an aircraft longitudinal separation of 4,000ft $(\approx 1,250$ metres $)$ in undisturbed air at an altitude of $30,000 \mathrm{ft}(\approx 9,150 \mathrm{~m})$ in standard atmospheric conditions (air density of $0.458 \mathrm{~kg} / \mathrm{m}^{3}$ ) at a speed of Mach 0.74 (cruise condition), at a mass of approximately 400,000 lb ( $\approx 182 \mathrm{e} 3 \mathrm{~kg}$ ) (182 tonnes). The lift required for level flight in this condition is $1,785,420 \mathrm{~N}$. The condition of Mach 0.74 at an altitude of 9,150 $\mathrm{m}$ results in a ground speed of $807 \mathrm{~km} /$ hour which is equivalent to $224 \mathrm{~m} / \mathrm{sec}$. The flight condition selected here corresponds to a point near the centre of the blue SAVE 1 region of figure 4 from [6].

We emphasise here that simulating UVLM for a longitudinal separation of 1250 metres involves unsteady simulation of many tens of thousands of wake vortex panels over many hundreds of time steps. Despite this huge computational task, the GPU implementation results in remarkably short wall-clock times of the order of 300 seconds.

The simulation runs were performed using a simplified model of the Boeing C-17 aircraft lifting surfaces derived from reference [14], and the Boeing C-17 on-line specification at reference [15]. We used the UVLM panel layout illustrated in figure 6. Since we have been unable to obtain more detailed information about the wing and tailplane aerofoil sections, twists, winglet design and anhedral angle, we have used a zero camber and flat planform design. This fact, along with the lack of account taken of vertical tail, fuselage and other appendages, means that good correspondence between the drag of the actual aircraft and the predictions made in these simulations is not expected.

The parametric study has been performed for a simulation frame rate of 40 frames per second. This results in roughly square wake panels.

Using all the above data, the Flexit UVLM calculations predict a lift of 1,783,660 N (181.820 tonnes), and a total induced drag of $64,823 \mathrm{~N}$ for the C-17 flying in isolation with a pitch attitude of 3.094 degrees ( 0.054 radians).

To cover the region presented in figure 33 of [6], we constructed a grid of 35 simulation cases in our parametric study. Figure 7 presents the locations of these 35 follower aircraft lateral positions.

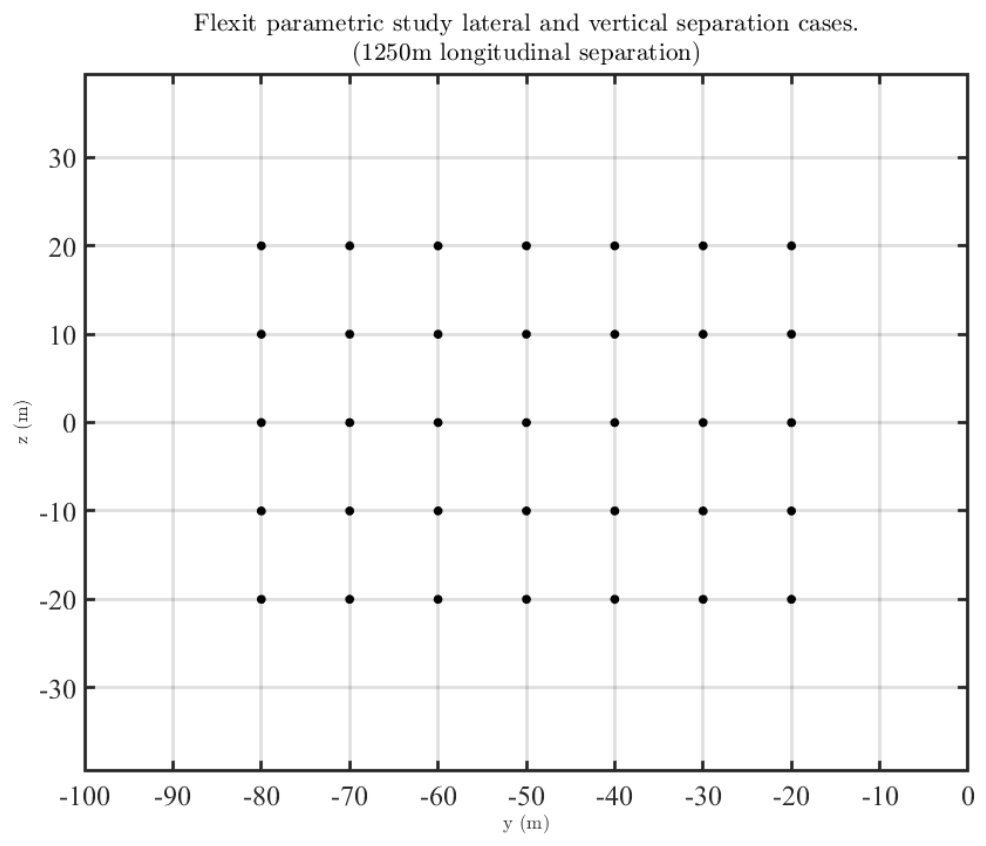

Fig. 7 Locations of the 35 follower aircraft lateral and vertical offsets considered in this parametric study.

For each case, we adopted the methodology presented in figure 5. We began by running Flexit with the follower aircraft at the baseline pitch attitude of 3.094 degrees. Typically this resulted in a lift prediction significantly higher than the baseline lift of 1,783,660 N. The follower aircraft pitch attitude was adjusted manually, and a second run was performed. The resulting lift was compared with the baseline value. This process was repeated until agreement between actual lift and required lift was obtained to an accuracy of 3 significanty figures. In this way, the pitch attitude of the follower aircraft is adjusted until the lift force equals the original aircraft weight. 
The drag reduction data from the parametric study is plotted as contours in figure 8 The figure illustrates that for all vertical offsets and for horizontal offsets outboard of 30 metres lateral displacement, the follower aircraft experiences a reduction in drag. The zero contour (corresponding to no benefit and no penalty) has been indicated in bold. The greatest reduction in induced drag is obtained when the follower aircraft flies approximately zero metres vertically offset and approximately 40 metres outboard offset relative to the lead aircraft.

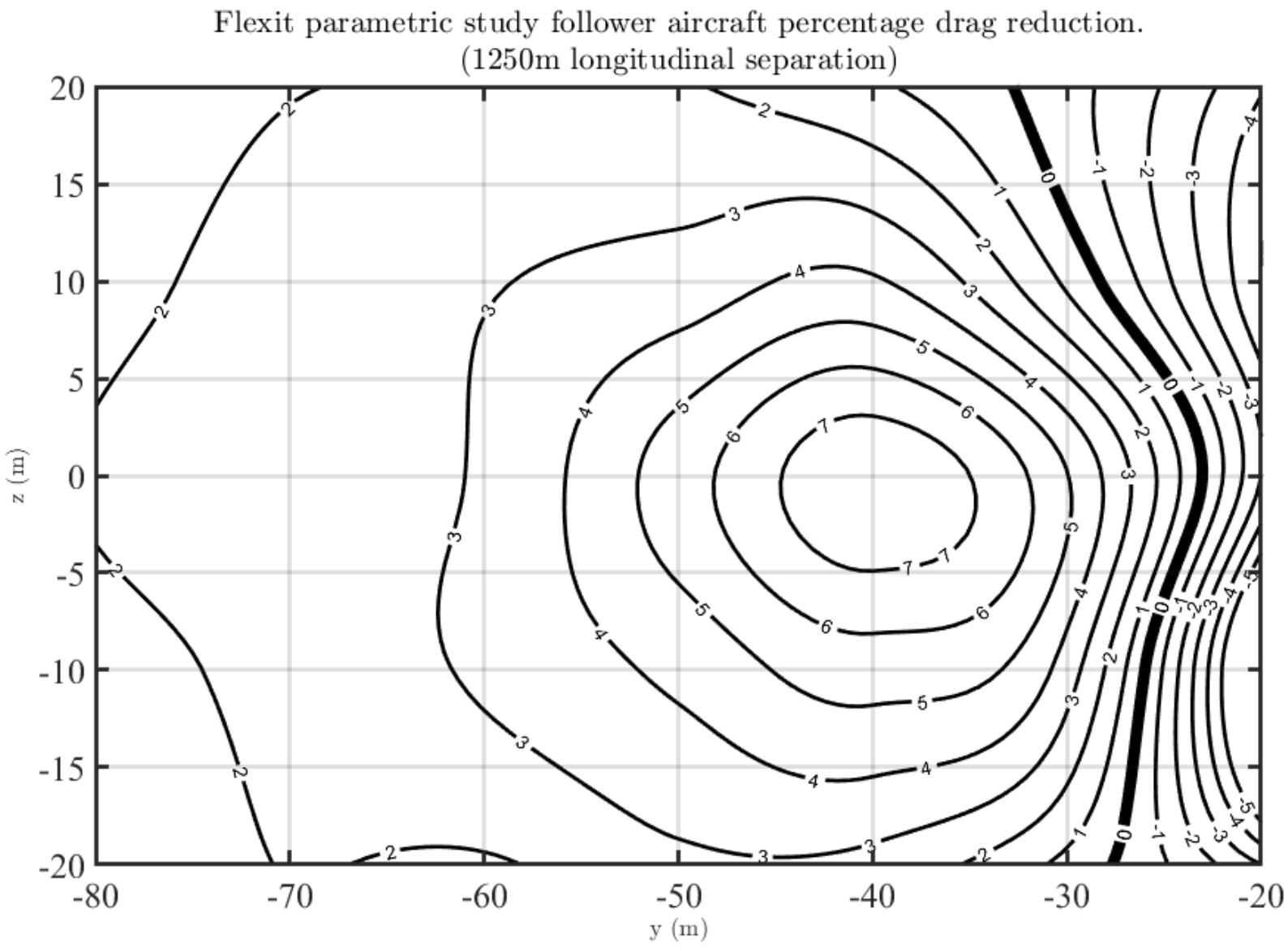

Fig. 8 Percentage reduction in induced drag predicted for follower aircraft in SAVE I formation (4,000 $f t$ longitudinal separation)

\section{E. Wake structure}

The predicted reductions in induced drag result from the follower aircraft flying in the wake of the lead aircraft. In particular, the upwash region of the trailing wing tip vortex. To gain some insight into the drag reduction mechanism, we computed the wake structure of the lead aircraft in the SAVE 1 configuration. This wake structure at a distances of $1250 \mathrm{~m}$ behind the lead aircraft (where the follower aircraft encounters the wake) is presented in figure 9 The figure is the vector field of induced velocities projected onto the $y z$-plane with contour plots of the vertical component of the induced velocity superimposed. The main wing tip vortex core appears to be located in a region 25 metres outboard, and slightly above the wing tip. Several smaller regions of vortical flow appear to exist nearer to the centreline and 10 to 15 metres below the main wing. These seem to result from mutual interaction between the tail plane tip vortex and the main wing wake sheet. 


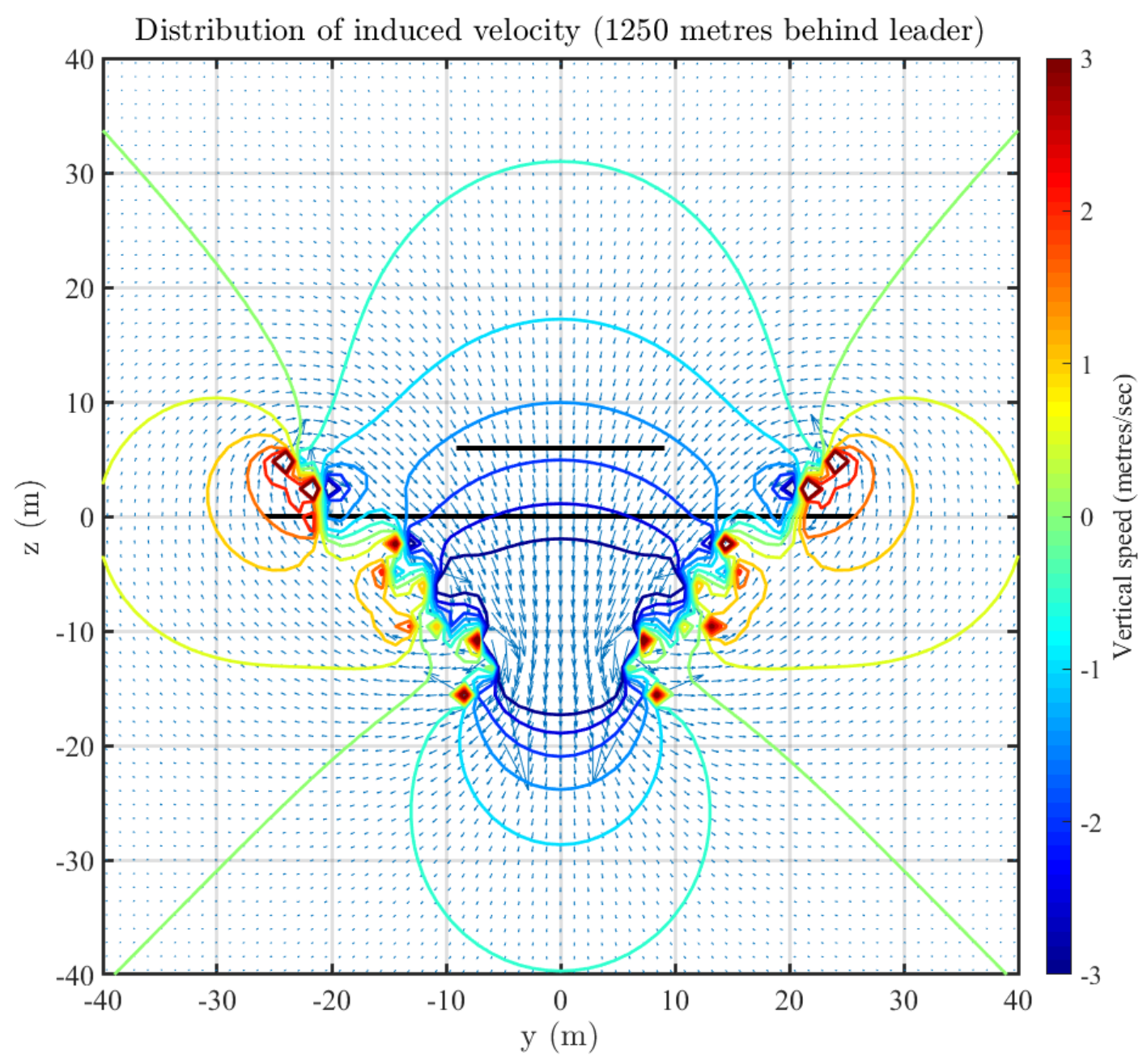

Fig. 9 Prediction of induced velocities $1250 \mathrm{~m}$ behind SAVE 1 lead aircraft

\section{Engine Performance Predictions}

To produce predictions of potential fuel (and hence cost) saving that results from wake surfing, the change in fuel consumption for each case in the parametric study is calculated based on the change in drag due to flight in the wake of the lead aircraft (for a fixed lift). To simplify the work, and to simplify the interpretation of results, we use the simple and well established Simulink ${ }^{\circledR}$ Turbofan Engine System from the Propulsion models of the Mathworks Aerospace blockset (see [8]). Figure 10 presents the Simulink ${ }^{\circledR}$ model used here.

The sea level static thrust has been set to 179947 newtons (based on the sea level static thrust quoted in reference [15]) for each of the four Pratt and Whitney PW2040 engines on the C-17 aircraft. A sea-level static thrust specific fuel consumption of $0.03348 \mathrm{~kg} /(\mathrm{Nhr})$ has been used (which is based on a value of $9.3 \mathrm{~g} /(\mathrm{kN} \mathrm{sec})$ published at [16]). The Simulink ${ }^{\circledR}$ model predicts a fuel mass flow rate for the C-17 flying in isolation of $0.3709 \mathrm{~kg} / \mathrm{sec}$ for each of the four PW2040 engines. Thus in this study we predict $f_{\text {ideal route }}^{n}=1.4836 \mathrm{~kg} / \mathrm{sec}$, for both $n=1$ (the lead aircraft) and $n=2$ (the follower aircraft).

As an illustrative example, table 1 presents the fuel consumption predictions made for the drag values of the leader and follower aircraft using the Simulink ${ }^{\circledR}$ model applied to case 4 of the SAVE 1 parametric study (1250 $\mathrm{m}$ x offset, 50 $m \mathrm{y}$ offset and $-20 \mathrm{~m} \mathrm{z}$ offset). It follows from the data presented in table 1 that the total fuel consumption of both aircraft flying independently is $0.3709 \times 8=2.967 \mathrm{Kg} / \mathrm{sec}$, whereas for the two aircraft flying in wake surfing formation, the total fuel consumption is $0.3709 \times 4+0.3624 \times 4=1.4836+1.4496=2.9332 \mathrm{Kg} / \mathrm{sec}$. Thus wake surfing results in a 


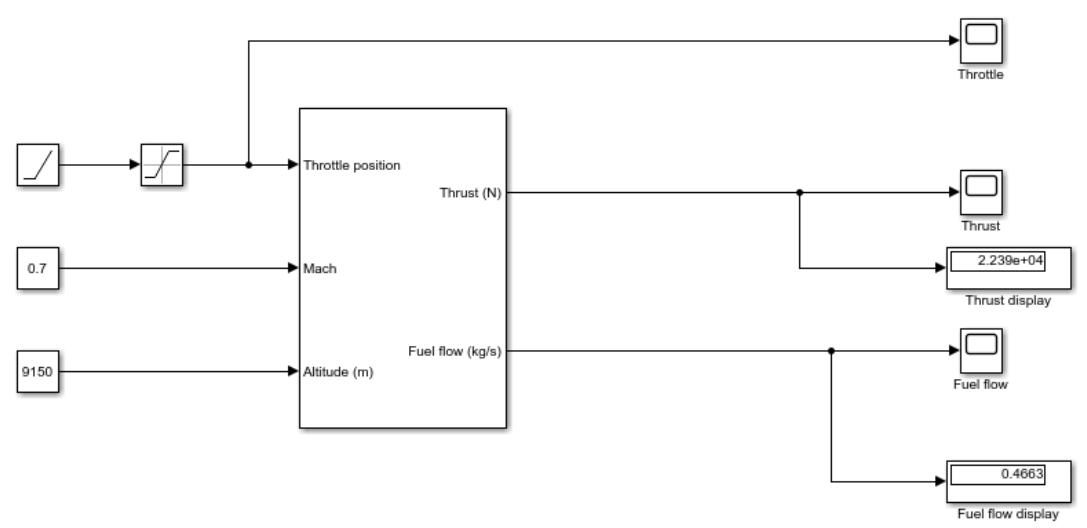

Fig. 10 Simple fuel consumption Simulink ${ }^{\circledR}$ model

net $0.034 \mathrm{Kg} / \mathrm{sec}$ or 1.14 percent fuel saving. In summary then, a reduction in drag of approximately 2.8 percent in the follower aircraft leads to a total net fuel saving of approximately 1.14 percent. Taking a price of aviation fuel of 0.57 Euros $/ \mathrm{kg}$, over a five hour wake surfing leg of a flight the saving is $0.57 \times 0.034 \times 3600 \times 5=348.84$ Euros. Figure 11 presents the data from the parametric study overlayed on figure 33 from [6].

\begin{tabular}{|lll|}
\hline Description & Leader & Follower \\
\hline \hline Lift & $1,783,660$ Newtons & $1,780,196$ Newtons \\
Drag & 64,823 Newtons & 63,005 Newtons \\
Percentage drag saving & $0 \%$ & $2.80 \%$ \\
Single engine required thrust & 16,205 Newtons & 15,751 Newtons \\
Throttle position & 0.436 & 0.422 \\
Model thrust & $16,273 \mathrm{Newtons}$ & $15,751 \mathrm{Newtons}$ \\
Single engine fuel consumption & $0.3709 \mathrm{Kg} / \mathrm{sec}$ & $0.3624 \mathrm{Kg} / \mathrm{sec}$ \\
Predicted percentage fuel saving & $0 \%$ & $2.29 \%$ \\
\hline
\end{tabular}

Table 1 Fuel consumption predictions for leader and follower aircraft for case 4 of the parametric study (1250 $m \mathbf{x}$ offset, $50 m$ y offset and $\mathbf{- 2 0} m z$ offset) at 40 frames per second.

\section{Discussion of results}

Here we compare the results of the parametric study with the results of the SAVE 1 flight tests presented in [6]. In this paper we have studied the benefit of wake surfing by comparing the performance of the trailing aircraft with the performance of the lead aircraft. It is because this comparison is relative that the absolute accuracy of the modelling is less important. This means that while we have used a simple inviscid drag formulation and a derived model for fuel consumption, the conclusions of the study are still valuable and meaningful. It is for this reason that we are justified in ignoring features such as fuselage drag, wing profile, empenage, engine nacelles and other design details.

Figure 33 from [6] presents a contour diagram of the trailing aircraft percentage fuel saving as a function of lateral and vertical offset relative to the lead aircraft vortex core. To estimate the position of the vortex core of the lead aircraft at the longitudinal position of the trailing aircraft predicted in our study, we consider figure 9 above. From this figure, it is clear that vortex structures are predicted on either side of the wake. They are both centred approximately 21 metres from the centreline, with the main one about level with the main wing and other smaller structures further inboard and below the aircraft. This is in general agreement with the data presented in [6].

Taking case 7 (-40 $\mathrm{m}$ y offset and $0 \mathrm{~m} \mathrm{z}$ offset), we predict a drag reduction of $7.69 \%$ and a fuel consumption saving of $5.88 \%$. Thus the relation between drag reduction and fuel saving is not one-to-one. This scaling factor results from 
SAVE 1 Follower Aircraft Fuel Saving as a function of Lateral and Vertical Separation.

Flight Trials vs Flexit Predictions (black)

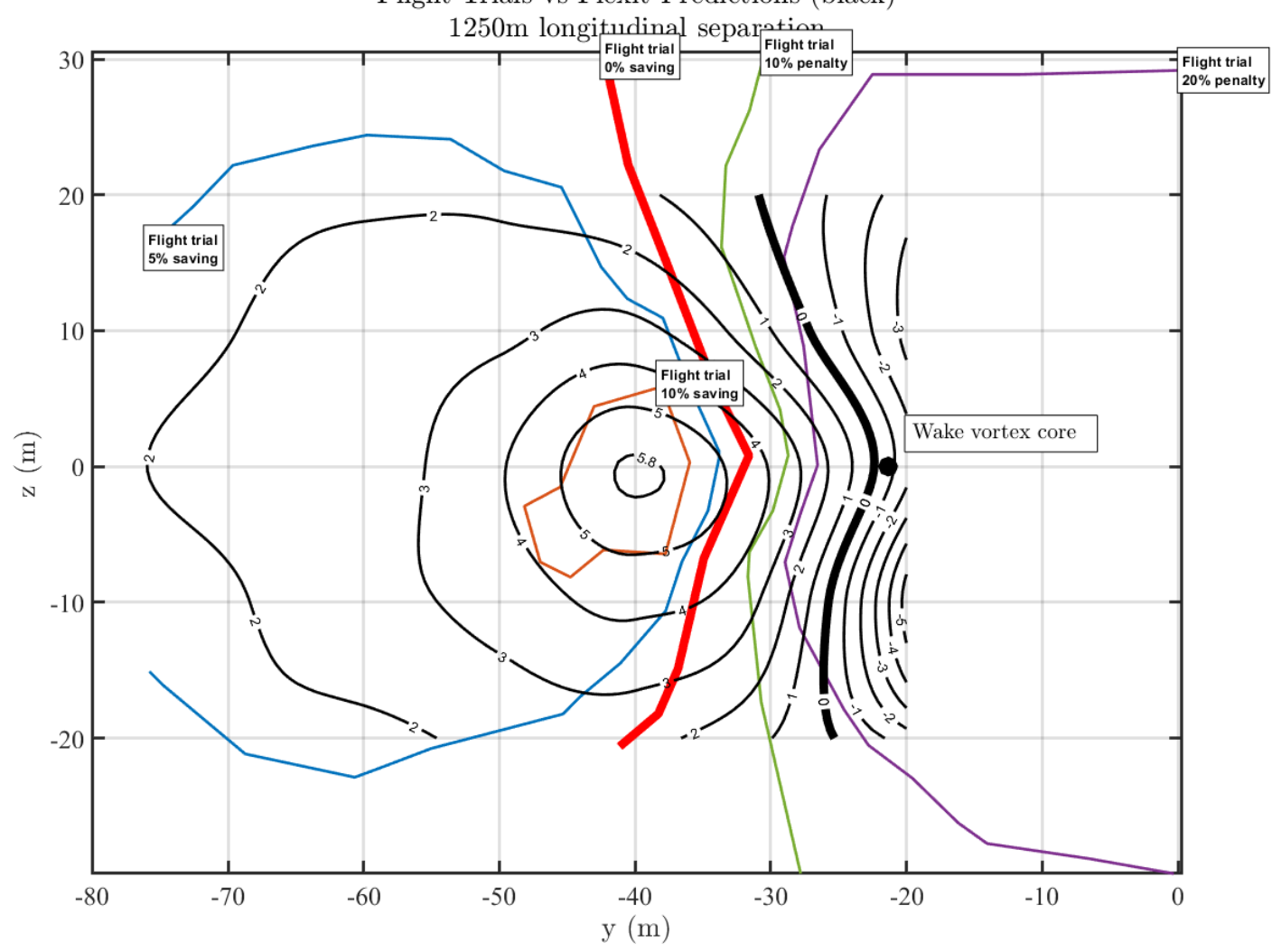

Fig. 11 Comparision of fuel savings predicted in the parametric study with the fuel savings experienced during the SAVE 1 flight trials

the Simulink ${ }^{\circledR}$ Turbofan Toolbox which is highly generic and simplistic model.

Figure 11 indicates that while the trends in fuel saving predicted in this parametric study are similar to those measured in the flight trials, the actual values are under-estimated by approximately 40\%. Part of this under-estimation may originate from the Simulink engine model.

While wake surfing is of benefit, for real operations the trailing aircraft will have a lower fuel burn, and therefore operate at a higher all-up weight for longer. This means that the overall saving will be reduced unless the fueling of the trailing aircraft takes into account the formation leg.

\section{Conclusions and future work}

We have presented a medium-fidelity analysis methodology to predict the potential fuel burn benefit from wake surfing. This methodology takes advantage of the Flexit program that implements UVLM algorithms running on modest hardware using the massive parallelization of the GPU architecture. This makes it suitable for the modelling of the large scale evolution of the wakes within aircraft wake surfing formations. These formations may extend over many thousands of metres.

In this case we have considered a very specific formation that corresponds to the SAVE 1 flight trial carried out by USAF AFRL and this provides a unique opportunity to compare predictions with real-world data. This is a homogeneous formation of two C-17 military transport aircraft flying with 1250 metres of longitudinal separation.

The data is presented in the form of a contour diagram showing the benefit as a function of vertical and horizontal position of the follower aircraft relative to the lead aircraft. The drag benefits were converted to fuel saving by using the standard Matlab $^{\circledR}$ Turbofan Toolbox. While the shape of the predicted fuel saving map is in general agreement with the flight trials, the simulations consistently under-predict the benefit. 
In the future, we intend to spend time on the following initiatives:

1) Make a deeper analysis of how our simulation method compares with the methods presented in [2].

2) Perform a more in-depth inverstigation of the relationship between drag reduction fuel saving.

3) Simulate heterogenous wake formations (with different types of aircraft in different configurations).

4) Model the aeroelastic behaviour of the trailing aircraft in both steady state cruise conditions and during transient wake crossing manoeuvres.

5) Estimate the drag penalty and associated fuel burn penalty associated with correcting the rolling moment experienced by the trailing aircraft.

6) Repeat the simulations with more accurate geometrical description of the lifting surfaces.

\section{Acknowledgements}

The authors would like to acknowledge the support of the Advanced Vehicle Technology Working Group 279 of the NATO Collaborative Support Office, as well as ATI and Innovate UK.

\section{References}

[1] Zhang, Q., and Liu, H. H. T., "Aerodynamics Modeling and Analysis of Close Formation Flight," American Institute of Aeronautics and Astronautics, Journal of Aircraft, 2017, pp. 2192-2204.

[2] Slotnick, J. P., Clark, R. W., Friedman, D. M., Yadlin, Y., Yeh, D. T., Carr, J. E., Czech, M. J., and Bieniawski, S. W., "Computational Aerodynamic Analysis for the Formation Flight for Aerodynamic Benefit Program," AIAA SciTech 2014, AIAA, 2014.

[3] Flanzer, T. C., Bieniawski, S. R., and Blake, W. B., "Operational Analysis for the Formation Flight for Aerodynamic Benefit Program,” 52nd Aerospace Sciences Meeting, AIAA SciTech Forum, National Harbour, Maryland, USA, 2014.

[4] Halaas, D. J., Bieniawski, S. R., Whitehead, B. T., and Flanzer, T., "Formation Flight For Aerodynamic Benefit Simulation Development and Validation," 52nd Aerospace Sciences Meeting, edited by AIAA, AIAA SciTech Forum, American Institute of Aeronautics and Astronautics, National Harbour, Maryland, USA, 2014.

[5] Bissonnete, W., and Bramesfeld, G., "Effects of Wake Shapes on High-Lift System Aerodynamic Predictions," Aerospace, Vol. 4, No. 2, 2017.

[6] Bieniawski, S. R., Clark, R. W., Rosenzweig, S. E., and Blake, W. B., "Summary of Flight Testing and Results for the Formation Flight for Aerodynamic Benefit Progam,” AIAA SciTech, 52nd Aerospace Sciences Meeting, Vol. 1457, AIAA, AIAA, 2014.

[7] Blake, W. B., Bieniawski, S. R., and Flanzer, T. C., "Surfing aircraft vortices for energy," The Journal of Defense Modeling and Simulation: Applications, Methodology, Technology, Vol. 12, 2013, pp. 31-39.

[8] Mathworks, "Matlab Turbofan Toolbox user manual," , 2018. URL https://uk.mathworks.com/help/aeroblks/ turbofanenginesystem.html

[9] Fleischmann, D., Lone, M., Weber, S., and Sharma, A., "Fast Computational Aeroelastic Analysis of Helicopter Rotor Blades," Scitech 2018, edited by A. I. of Aeronautics and Astronautics, American Institute of Aeronautics and Astronautics, American Institute of Aeronautics and Astronautics, 2018.

[10] Katz, J., Plotkin, A., Low Speed Aerodynamics, Cambridge University Press, 2001.

[11] Karamcheti, K., Principles of Ideal-Fluid Aerodynamics, Robert E. Krieger Publishing Company, Huntington, New York, 1980.

[12] “Official NVIDIA CUDA Web site,”, 2017. URL http://www.nvidia.com/object/cuda_home_new.html

[13] Melin, T., Isikveren, A. T., and Friswell, M. I., "Induced-Drag Compressibility Correction for Three-Dimensional Vortex-Lattice Methods," Journal of Aircraft, Vol. 47, 2010.

[14] Tyler, J. R., and Instan-Tanious, “C-17,” Technical report, Virginia Technical University, 2009.

[15] Boeing, "C-17 Globemaster III technical specification," , 2017. URL http://www.boeing.com/defense/c-17globemaster-iii/

[16] Wikipedia, “Thrust-specific fuel consumption,”, 2018. URL https://wikivisually.com/wiki/Thrust-specific_ fuel_consumption 
2019-01-08

\section{Analysis of wake surfing benefits using a fast unsteady vortex lattice method}

Fleischmann, Dominique

AIAA

Fleischmann D, Lone M. (2019) Analysis of wake surfing benefits using a fast unsteady vortex lattice method. Proceedings of the AIAA Scitech 2019 Forum, 7-11 January 2019, San Diego, CA, USA

https://arc.aiaa.org/doi/book/10.2514/MSCITECH19

Downloaded from Cranfield Library Services E-Repository 